\title{
Earlier Development of Limb Ulcers, Digital Bone Infarction and Pulmonary Hypertension in a Patient with SLE- A Rare Case Report
}

\author{
M HEZBULLAH $^{\mathrm{a}}$, SR CHAKRABARTY ${ }^{\mathrm{b}}, \mathrm{S}^{\mathrm{S}}$ SULTANA $^{\mathrm{c}}$, MH TOHIN $^{\mathrm{d}}, \mathrm{K}^{\mathrm{N}}$ NAHAR $^{\mathrm{e}}$
}

\begin{abstract}
Summary:
Systemic lupus erythematosus (SLE) is a multiorgan autoimmune disease in which skin is involved in up to $85 \%$ of cases. Lower extremity ulcers are an infrequent but disabling complication of SLE. Critical peripheral ischemia (CPI) is also an uncommon but potentially devastating feature of SLE. We reported a case of SLE who presented with multiple ulcers along with digital infarcts of upper and lower limbs. She had features of digital bone infarct of left hand and right foot. She also had features of pulmonary interstitial involvement with pulmonary hypertension. All
\end{abstract}

\section{Introduction:}

Connective tissue disorders (CTD), often termed collagen vascular diseases includes a number of related inflammatory conditions like Rheumatoid arthritis, Systemic lupus erythematosus, Systemic sclerosis, Localized scleroderma, Sjogren's syndrome, Dermatomyositis, Polymyositis and Mixed connective tissue disease. ${ }^{1}$ Systemic lupus erythematosus (SLE) is a multiorgan autoimmune disease of unknown etiology that may have many clinical manifestations. The skin is involved in up to $85 \%$ of Systemic lupus erythematosus cases and may be the only organ involved in Cutaneous lupus erythematosus (CLE). ${ }^{2}$ Lower extremity ulcers are an infrequent but disabling complication of long-

a. Dr. Muhammad Hezbullah, Associate Professor (Medicine), Sylhet MAG Osmani Medical College, Sylhet.

b. Dr. Shishir Ranjan Chakrabarty, Associate Professor (Medicine), Sylhet MAG Osmani Medical College, Sylhet.

c. Dr. Sharifa Sultana, Assitant Professor (Pharmacology), Jalalabad Ragib Rabeya Medical College, Sylhet.

d. Dr. Manwarul Haque Tohin, Indoor medical officer (Medicine), Sylhet MAG Osmani Medical College Hospital, Sylhet.

e. Dr. Kamrun Nahar, Senior Medical Officer and Director (in charge), Institute of Nuclear Medicine \& Allied Sciences, Sylhet.

Address of Correspondence: Dr. Muhammad Hezbullah, Associate Professor (Medicine), Sylhet MAG Osmani Medical College Sylhet, Mobile: 01717020500,Email: mhezbullah@yahoo.com

Received: 19 October 2016

Accepted: 7 June 2017 of these features were found within three months of disease onset which is very rare. She was treated with intravenous Methylprednisolone followed by oral Prednisolone, Hydroxychloroquinine, Azathioprine, Diuretic, Bosentan, Aspirin and Nifedipine in combinaton. Later warfarin was added after one month. She showed significant improvement after three months of treatment.

Key words: Limb ulcer, bone infarct, pulmonary hypertension, Systemic lupus erythematosus.

(J Bangladesh Coll Phys Surg 2017; 35: 150-154)

standing connective tissue diseases. ${ }^{3}$ Critical peripheral ischemia (CPI) is an uncommon but potentially devastating feature of SLE although little is published as to its prevalence and management. The pathogenesis of critical ischemia in SLE is complex and multifactorial, involving capillary and arteriolar vasospasm associated with Raynaud's phenomenon, active large and small vessel vasculitis, micro-vascular thrombosis and emboli associated with antiphospholipid and accelerated atherosclerosis. ${ }^{4}$ Raynaud's phenomenon describes temperature-sensitive, digital vasospasm leading to pale and consecutively cyanotic skin mostly limited to the digits, occurring in 3\% to $5 \%$ in the general population. Besides the uncomplicated primary Raynaud's phenomenon, the secondary form occurring in connective tissue disease presents more severely, with potentially disabling ulceration or tissue necrosis. ${ }^{5}$ Although Raynaud's phenomenon is fairly common in patients with SLE, digital ulcers are quite rare. ${ }^{6}$

\section{Case Report:}

A 24 yrs old female normotensive, non-diabetic patient hailing from Sunamgonj, Sylhet was admitted in medicine department of Sylhet MAG Osmani medical college hospital on $3^{\text {rd }}$ March, 2016 with the complaints of multiple ulcers in both upper and lower limbs for 3 months, pain in multiple joints for 3 months, weakness and generalized muscular pain for 2 months. At first she noticed few blisters on her right leg which ruptured and turned into an ulcer. Gradually she developed 
multiple ulcers on her both legs and hands which were not healing, painful, gradually increasing in size with serous discharge (Figure 1).

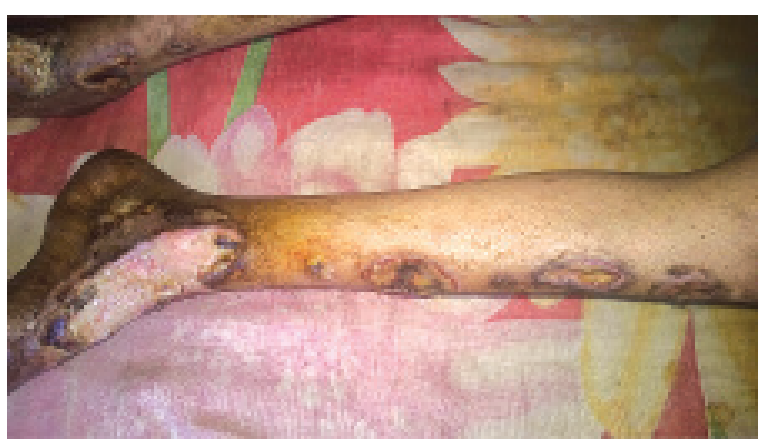

Fig.-1: Leg ulcers of left leg

She also noticed gradual blackening of tips of her toes and fingers with pain which used to increase on cold water exposure. Her joint pain involved both small and large joints of upper and lower limbs with swelling persisting throughout the day with morning stiffness. She also complained of gradual loss of scalp hair, anorexia and weight loss for the last 3 months. On query, the patient told that she developed cough for the last 2 months which is dry, not associated with any blood and not related with any posture. She gave no history of fever, shortness of breath, oral ulcer, photosensitivity, convulsion, abdominal pain, dry mouth, dry eye, dysphagia, tightening of hand and foot. She gave no history of spontaneous abortion. She took some painkiller and antibiotics during her period of illness. She is nonsmoker, nonalcoholic and came from a poor family with none of her family member having similar type of illness. On general examination patient was illlooking, emaciated with hyper pigmented area over the forehead, cheek and nasal bridge sparing the nasolabial folds. Mild anemia, alopecia, edema were found with pulse-90/min, BP-100/70 mm of $\mathrm{Hg}$ and temparature$98.8^{0} \mathrm{~F}$. Multiple ulcers were present over the skin of tibia, medial malleolus, lateral malleolus, dorsum of foot, index and ring fingers of both hands. The ulcers were variable in size, shape and depth with largest one measuring about $7 \mathrm{~cm} \times 4 \mathrm{~cm} \times 3 \mathrm{~cm}$ and smallest one measuring $2.5 \mathrm{~cm} \times 1 \mathrm{~cm} \times 2 \mathrm{~cm}$, muscle depth, margin is sloping, floor covered with granulation tissue with scanty serous discharge, slightly tender and surrounding skin is normal. On musculoskeletal system examination interphalangeal joints, metacarpophalangeal joints of both hands were tender, mildly swollen with tapering of fingers, necrosis and ulceration, gangrenous changes of finger tips with atrophy of pulp and loss distal phalanx of little finger of left hand (Figure 2).

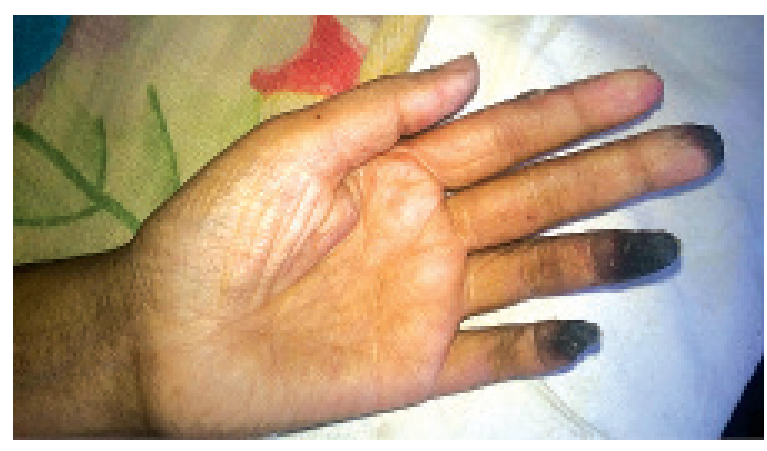

Fig.-2: Digital gangrene of left hand

There was dorsal guttering with mild wasting of both thenar and hypothenar muscles of both hands. All the metatarsophalangeal and interphalangeal joints are tender and mildly swollen. There were gangrenous changes of the toes of both feet. Both wrists, ankle and knee joints were tender and mildly swollen. On examination of precordium palpable $\mathrm{P} 2$, left parasternal heave and loud pulmonary component of $2^{\text {nd }}$ heart sound were found. On abdominal examination mild tender hepatomegaly was found. On examination of chest, crepitations were found in both apical areas and left base. Other system examinations were unremarkable. Investigations revealed following findings: $\mathrm{Hb} \%-9.7$ $\mathrm{gm} / \mathrm{dL}, \mathrm{ESR}-80 \mathrm{~mm}$ in $1^{\text {st }}$ hour, Urine R/M/E- protein+ pus cell 7-8/HPF, $24 \mathrm{hrs}$ total urinary protein- .28gm/ $24 \mathrm{hrs}$, serum creatinine-.63 mg/dL, RA testPositive(20.7 iu/ml), serum ANA- strongly positive (116.9 iu/ml), serum anti-ds DNApositive $(94.50 \mathrm{iu} / \mathrm{ml})$, serum c-ANCA-negative $(<1.47$ $\mathrm{U} / \mathrm{ml})$, serum $\mathrm{p}$-ANCA-negative $(<1.47 \mathrm{U} / \mathrm{ml})$, serum anti RNP- negative, antiphospholipid antibodynegative, anticardiolipin antibody-negative, lupus anticoagulant- negative, ECG- right axis deviation, CXR $\mathrm{P} / \mathrm{A}$ view- RV type cardiomegaly with fullness of pulmonary conus, colour doppler echocardiographyright sided pressure overload with moderate pulmonary hypertension(PASP-58 mm Hg) and mild pericardial effusion, X-ray hand- periarticular osteopenia and loss of terminal phalanx of little finger of left hand (Figure 3), X-ray of foot- periarticular osteopenia with tapering of terminal phalanx of right great toe. 


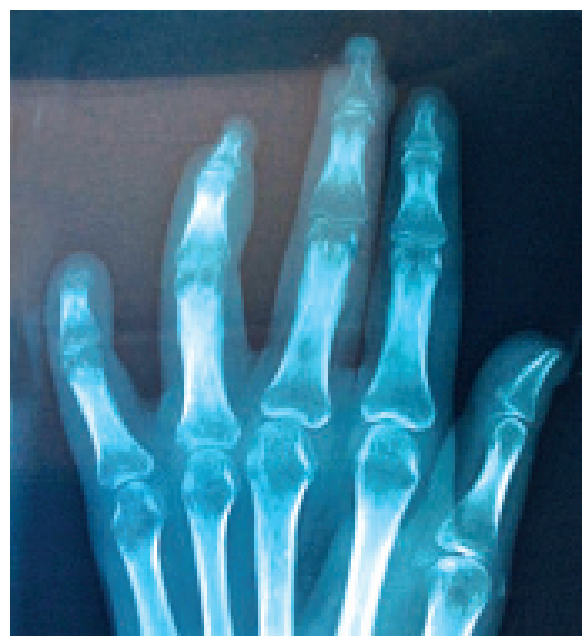

Fig.-3: X ray left hand showing loss of terminal phalanx of little finger

Biopsy of leg ulcer- Non specific inflammation, HRCT of chest- parenchymal scarring with fibrotic strands of upper lobes, left basal pneumonitis and pericardial effusion. Duplex study of both upper limb vessels showed no significant obstruction in radial, ulnar, brachial and axillary arteries. Duplex study of both lower limb vessels showed mild $(<20 \%)$ luminal narrowing at posterior tibial and dorsalis pedis arteries. Small digital arteries could not be evaluated by duplex study. Finally the case was diagnosed as "Systemic Lupus Erythematosus (SLE) with pulmonary hypertension and Raynaud's phenomenon" depending on diagnostic criteria of SLE. The patient was treated initially with intravenous Methylprednisolone $1 \mathrm{gm} /$ day for 3 days, followed by daily $100 \mathrm{mg}$ IV infusions for two weeks. Then oral Prednisolone $1 \mathrm{mg} / \mathrm{kg} /$ day was given with reduction of dose by $10 \%$ every two weeks until $20 \mathrm{mg} /$ day which was maintained. In addition Hydroxychloroquinine $200 \mathrm{mg} 12$ hourly and Azathioprine $50 \mathrm{mg} 12$ hourly was also given. Cyclophosphamide was avoided because the patient is married for one year and yet to conceive. Diuretic and Bosentan was given orally for pulmonary hypertension. Aspirin and Nifedipine was given for Raynaud's phenomenon. Amoxycillin and paracetamol was given for ulcer infection along with daily dressing of ulcers and digital gangrene. Orthopedics and plastic surgery consultation was taken about the management of ulcers and digital gangrene. Patient was discharged with oral medication and follow up after one month. On follow up visit patients joint manifestations subsided and ulcers were found to almost healing, alopecia reversed but digital gangrene was not improving. Then we started warfarin $5 \mathrm{mg}$ daily orally after performing baseline prothombin time along with running medications with advice to come back again after one month. After one month of starting warfarin digital infarcts were found to be healing and improved a lot.

\section{Discussion:}

Despite being a common occurrence in connective tissue diseases, digital ulcer and gangrene development is only occasionally seen in patients with SLE. The most common cause of digital gangrene associated with SLE is antiphospholipid antibody syndrome(AAS) and SLE is the leading cause of secondary AAS. ${ }^{7}$ The prevalence of AAS in SLE is reported to be $30 \%$ to $50 \% .{ }^{8}$ Some patients with AAS develop digital ischemic symptoms resulting in gangrene of digits. The prevalence is reported to be 3.3-7.5\% of AAS patients. ${ }^{9}$ Skin ulcers are also common and are often seen on the pretibial and ankles. Cutaneous gangrene was found in $19 \%$ of AAS patients and cutaneous necrosis in $3 \% .{ }^{10}$ Similar involvement of digital gangrene and skin ulcers in typical site was found in our patient. But antiphospholipid antibody, anticardiolipin antibody and lupus anticoagulant were negative in our patient. These antibodies were also found negative in 3 out of 7 patients with critical peripheral ischemia in diagnosed SLE patient. ${ }^{4}$ Another contributing factor to digital gangrene in SLE could be atherosclerotic changes in the arteries. Increasing attention has been drawn to late complications of lupus-like atherosclerotic vascular disease. ${ }^{11}$ But our patient did show only minor $(<20 \%)$ obstruction in her dorsalis pedis and posterior tibial arteries by duplex study. This may be due to the fact that the disease in our patient started only three months back. Liu et al. demonstrated that duration of disease, the presence of Raynuad's phenomenon and high serum CRP levels were determinants of digital ulcer development in a cohort of 2600 lupus patients. ${ }^{12}$ But our patient presented with a short history though she had Raynaud's phenomenon and high CRP. Jeffery et al. reviewed the records of a cohort of 485 
patients with SLE and found the mean duration of disease before onset of critical ischemia was $9.4 \mathrm{yrs}$ with mean age at onset $31.5 \mathrm{yrs} .{ }^{4}$ But our patient presented with digital ischemic symptoms at diagnosis and patient is only $24 \mathrm{yrs}$ of age.

Pulmonary arterial hypertension $(\mathrm{PAH})$ is defined as a mean pulmonary artery pressure $\geq 25 \mathrm{~mm} \mathrm{Hg}$ and pulmonary capillary wedge pressure of $\leq 15 \mathrm{~mm} \mathrm{Hg}$. PAH is a severe manifestations of many of the seropositive connective tissue diseases. It has long been recognized as a manifestation of systemic sclerosis and systemic lupus erythematosus. PAH may be an underrecognized manifestation of SLE. ${ }^{13}$ Vascular pathologic findings in patients with SLE associated PAH include plexiform lesions, muscular hypertrophy and intimal proliferation. ${ }^{8}$ Systematic review of patients with SLE indicate that the incidence of pulmonary hypertension is $0.5 \%$ to $23.3 \%$ and that the diagnosis of pulmonary hypertension occurs 4.9 years to 10.7 years after the initial diagnosis of SLE. Maehara et al. reported a case in which pulmonary hypertension was diagnosed 18 years after the diagnosis of SLE. ${ }^{14}$ But when we look at our case the patient presented with manifestations of pulmonary hypertension at the time of initial diagnosis of SLE. Osteonecrosis or bone death caused by ischemia, in SLE is not uncommon. Osteonecrosis occurs with prominent symptoms at a rate of $3 \%$ to $30 \%$. Osteonecrosis in SLE is largely secondary to avascular necrosis which by definition occurs in the epiphysis or subarticular bone. Osteonecrosis of the metaphysis or diaphysis of the bone is referred to as bone infarction and is rarely seen in SLE. ${ }^{15}$ In our patient bone infarction was found in tip of great toe of right foot and distal phalanx of left little finger.

The treatment of digital ulcers in patients with SLE includes Epoprostenol(Iloprost) infusion, bosentan and immunosuppressive therapy (Cyclophosphamide and pulse Methylprednisolone). ${ }^{6}$ Unfortunately Epoprostenol(Iloprost) was not available, so could not be given to our patient. Cyclophosphamide was avoided intentionally because patient is married for one year and yet to conceive. Instead pulse Methylprednisolone followed by oral Prednisolone, Aspirin, Hydroxychloroquine, Azathioprine, Nifedipine and Bosentan was used in combination which was later added with warfarin. Bosentan's efficacy for digital ulcers in Systemic sclerosis has been well recognized. It might be an alternative treatment for refractory digital ulcers in SLE. ${ }^{11}$ Our patient's limb ulcers was found to be healed almost completely after 3 months of above therapy. Her digital gangrene was also improving after adding of Warfarin.

\section{Conclusion:}

We have reported this case because the patient presented with Raynaud's phenomenon along with digital gangrene, limb ulcers, bone infarction and pulmonary hypertension in combination at the time initial diagnosis. These types of presentation at diagnosis of SLE are very rare.

\section{Acknowledgement:}

Dr. Shaek Aziz chowdhury, Managing director, Popular medical centre, Sylhet.

\section{References:}

1. Dabiri G, Falanga V. Connective tissue ulcers. J Tissue Viability 2013;22(4):92-102.

2. Uva L, Miguel D, Pinheiro C, Freitas JP, Gomes MM and Filipe P. Cutaneous manifestations of Systemic Lupus Erythematosus. Autoimmune Diseases 2012, Article ID 834291, 15 pages. Doi: 10.1155/2012/834291.

3. Santos G, Joao A, Sousa L. Leg ulcers in antiphospholipid syndrome secondary to a systemic lupus erythematosus treated with intravenous immunologlobulin. J Dermatol Case Rep $2014 ; 2: 38-41$

4. Jeffery RC, Narshi CB, Isenberg DA. Prevalence, serological features, response to treatment and outcome of critical peripheral ischaemia in a cohort of lupus patients. Rheumatology 2008;47:1379-1383.

5. Fries R, Shariat K, Wilmowsky HV, Bohm M. Sildenafil in the treatment of Raynaud's phenomenon resistant to vasodilatory therapy. Circulation 2005;112:2980-2985.

6. KÜÇÜK A, BACACI S, SOLAK Y, TUNÇ R. Digital ulcers as a rare complication of Systemic Lupus Erythematosus: A case report. Arch Rheumatol 2014;29(1):53-56.

7. Mills JA. Systemic lupus erythematosus. N Engl J Med 1994;330:1871-9.

8. Changal KH, Sofi F, Altaf SS, Raina A, Raina AH. ANA negative Systemic Lupus Erythematosus leading to CTEPH, TTP-like thromobocytopenia and skin ulcers. Case reports in Rheumatology. Volume 2016, Article ID 4507247, 5 pages.

9. Asherson RA, Frances C, Iaccarino L, Khamashta MA, Malacarne F, Piette JC, et al. The antiphospholipid antibody 
syndrome: diagnosis, skin manifestations and current therapy. Clin Exp Rheumatol 2006;24:S46-S51.

10. Algre VA, Gastineau DA, Winkelmann RK. Skin lesions associated with circulating lupus anticoagulant. Br J Dermatol 1989;120:419-429.

11. Nagai $\mathrm{Y}$ et al. Digital gangrene in Systemic Lupus Erythematosus. Acta Derm Venereol 2009;89:398-401.

12. Liu A, Zhang W, Tian X, Zhang X, Zhang F, Zeng X. Prevalence, risk factors and outcome of digital gangrene in 2684 lupus patients. Lupus 2009;18:1112-8.
13. Johnson SR, Granton JT. Pulmonary hypertension in systemic sclerosis and systemic lupus erythematosus. Eur Respir Rev 2011;20:277-286

14. Maehara Y, Kawasaki T, Miki S, Enoki Y, Kamitani T. Natural course of pulmonary hypertension due to systemic lupus erythematosus: A case report. Int J Clin Case Stud 2015;1:105

15. Lederhandler M, Valins W, Zoghbi Z, Grossman ME. Leg ulcers in systemic lupus erythematosus associated with underlying dystrophic calcinosis and bone infarcts in the absence of antiphospholipid antibodies. Journal of American Academy of Dermatology Case Reports 2016;2:164-7. 